\title{
Sergeia podlipaevi gen. nov., sp. nov. (Trypanosomatidae, Kinetoplastida), a parasite of biting midges (Ceratopogonidae, Diptera)
}

\author{
Milena Svobodová, ${ }^{1}$ Lenka Zídková, ${ }^{1}$ Ivan Čepička, ${ }^{2}$ Miroslav Oborník, ${ }^{3}$ \\ Julius Lukešs ${ }^{3,4}$ and Jan Votýpka, \\ ${ }^{1}$ Department of Parasitology, Faculty of Science, Charles University, Prague, Czech Republic \\ ${ }^{2}$ Department of Zoology, Faculty of Science, Charles University, Prague, Czech Republic \\ ${ }^{3}$ Biology Centre, Institute of Parasitology, Czech Academy of Sciences, České Budějovice \\ (Budweis), Czech Republic \\ ${ }^{4}$ Faculty of Biology, University of South Bohemia, České Budějovice (Budweis), Czech Republic
}

Correspondence

Jan Votýpka

vapid@natur.cuni.cz

\section{INTRODUCTION}

Trypanosomatids are virtually omnipresent and are among the most successful of parasites. Discussion about the origin of trypanosomatids remains open (Simpson et al., 2006) and the recent discovery of flagellates that morphologically resemble extant trypanosomatids in $\sim 100$ million-year-old amber-embedded biting midges, sand flies and other insects (Poinar \& Poinar, 2004, 2005) provides additional support

\footnotetext{
Abbreviations: AM, abdominal midgut; DPI, days post-infection; gGAPDH, glycosomal glyceraldehyde-3-phosphate dehydrogenase; MT, Malpighian tubes; OTUs, operational taxonomic units; RAPD, random amplified polymorphic DNA; SL RNA, spliced-leader RNA.

The GenBank/EMBL/DDBJ accession numbers for the 18S, 5S and SL RNA and partial gGAPDH gene sequences of Sergeia podlipaevi gen. nov., sp. nov. are D0394362, D0394363, DQ394364 and DO394365, respectively.

A table detailing the GenBank accession numbers for the 18S, 5S and SL RNA and partial gGAPDH gene sequences analysed in this study and the composition of the final combined dataset is available as Supplementary Table S1 in IJSEM Online. Two figures, a representative RAPD gel and a phylogenetic tree inferred from the dataset of all the RAPD patterns, are also available as supplementary material.
}

for the notion that insects have been the primary hosts for these parasites. Trypanosomatids may have infected other invertebrates, plants and vertebrates including humans at a later stage either by contamination or through a bite (Wallace, 1966; Vickerman, 1994). Such an extremely long cohabitation could result in a co-evolution, with perhaps hundreds of thousands of flagellate species occurring in insects (Stevens, 2001). Nevertheless, recent literature includes only about a thousand descriptions of monoxenous trypanosomatids from insects, of which no more than three hundred can be considered as recognized species (Podlipaev, 1990, 2000).

The previously widely applied 'new host-new parasite' paradigm apparently does not reflect the reality (Podlipaev, 1990, 2000) and cannot be used as a basis for species description. Although we cannot exclude the existence of stenoxenous species, data obtained during two extensive studies into the diversity of monoxenous trypanosomatids parasitizing dipteran and hemipteran insects in Russia and Costa Rica (Podlipaev et al., 2004a; Westenberger et al., 2004) clearly show that at least some trypanosomatid species may infect a wide range of insect hosts, in some cases even 
spanning different host orders. At the same time, one insect host can be infected with more than a single flagellate species (Podlipaev et al., 2004a).

Classical trypanosomatid taxonomy is based on a narrow set of morphotypes that are characteristic for a given genus, such as cell shape and dimensions and the relative position of the nucleus and the kinetoplast (Hoare \& Wallace, 1966). Yet these morphotypes can be extensively modified by environmental factors or by introduction into culture (Podlipaev, 2000; Momen, 2001; Yurchenko et al., 2006). Species determination is thus hampered by the dearth of morphological features, with those few available being highly variable. Two decades ago, Wallace et al. (1983) proposed the utilization of other informative characters, such as differences in biochemistry, nutrition and ultrastructure. Ensuing research has indeed identified differences in biochemical features, including the composition of cellsurface polysaccharides, sialidases and proteolytic enzymes and the content and number of acidocalcisomes. Some of these features are sufficient to distinguish between strains and species and many recent species descriptions have been based on one, or on a combination, of these criteria (Abreu Filho et al., 2001; d'Ávila-Levy et al., 2004; Miranda et al., 2004; Santos et al., 2005). Unfortunately, these features fail to discriminate between genera.

The discriminatory power of molecular markers appears to be significantly higher. RNA sequences, such as smallsubunit rRNA (18S rRNA), 5S rRNA, spliced-leader RNA (SL RNA), and protein-coding genes, such as glycosomal glyceraldehyde-3-phosphate dehydrogenase (gGAPDH) and cytosolic heat-shock protein 90 (cHSP90), have proved very important for our understanding of the evolution of medically important and widespread trypanosomatids of the genera Trypanosoma and Leishmania, the relationships of which cannot be addressed using morphological criteria (Fernandes et al., 2001; Stevens et al., 2001; Simpson et al., 2002, 2006; Hamilton et al., 2004). Similarly, we anticipate that molecular phylogenies based on these and other genes will play a key role in revising the outdated taxonomy of trypanosomatids as a whole. All studies performed so far, even with a limited number of analysed sequences, have revealed major discrepancies between the morphology- and sequence-based systems (Hollar et al., 1998; Merzlyak et al., 2001; Momen, 2001). The number of species representing all currently recognized genera of insect monoxenous trypanosomatids (Crithidia, Blastocrithidia, Herpetomonas, Wallaceina and Leptomonas) for which gene sequences are becoming available is growing and so is the disparity between the above-mentioned systems (Stevens et al., 2001; Podlipaev et al., 2004a, b; Westenberger et al., 2004; Yurchenko et al., 2006). As a matter of fact, none of these genera have survived as a monophyletic taxon and there is general agreement in the field that the recognition of genera among trypanosomatids urgently needs a major revision to reflect the data amassed by molecular taxonomy.
In this study, we present a description of a novel trypanosomatid flagellate isolated from two species of biting midges. This organism, Sergeia podlipaevi gen. nov., sp. nov., forms a new major clade in phylogenetic trees based on several molecular markers. The sequences of its $18 \mathrm{~S}$ rRNA, SL RNA, 5S rRNA and gGAPDH genes are so unrelated to those currently available in databases that we propose the creation of a new genus to accommodate this species. We feel that the concept of creating new taxonomic units solely on the basis of sequence data represents a necessary step towards a taxonomy that will reflect true relationships among trypanosomatids.

\section{METHODS}

Organisms. Several isolates of Sergeia podlipaevi gen. nov., sp. nov. were obtained from females of two species of biting midges Culicoides (Oecacta) festivipennis Kieffer, 1914 (syn. C. odibilis Austen, 1921) and Culicoides (Oecacta) truncorum Edwards, 1939 (syn. C. sylvarum Callot et Kremer, 1961). The insects were caught while they were attacking the nestlings of the common buzzard (Buteo buteo) in Milovicky forest in the vicinity of Mikulov, Southern Moravia, Czech Republic. Females of six Culicoides species captured from nests of the common buzzard in Milovicky forest and nests of the sparrow hawk (Accipiter nisus) in the suburbs of Prague were collected from May to July each year between 1998 and 2002 (see Table 1). Insects were collected using air-sucking miniature Centers for Disease Control traps without a light bulb which were placed at the level of the nests, $10-18 \mathrm{~m}$ above the ground. Trapped biting midges were dissected within 2 days under a stereomicroscope and their alimentary tracts were examined whole by light microscopy. When flagellates were noticed in the intestine, most frequently in the abdominal midgut (AM) or in the Malpighian tubes (MT), the alimentary tract was inoculated into a $2.5 \mathrm{ml}$ glass vial with rabbit blood agar (SNB-9) supplemented with $80 \mu \mathrm{g}$ gentamicin $\mathrm{ml}^{-1}$ and $10000 \mathrm{IU}$ penicillin $\mathrm{ml}^{-1}$ and kept at room temperature. Successfully established cultures were passaged and stored in a cryobank at the Department of Parasitology, Faculty of Science, Charles University, Prague, as strains ICUL/CZ/1999/CER1, ICUL/ CZ/2000/CER2, ICUL/CZ/2000/CER3, ICUL/CZ/2000/CER4, ICUL/ CZ/2001/CER6, ICUL/CZ/2001/CER7 and ICUL/CZ/2001/CER8 and studied further. The material remaining on the original slide was air-dried and fixed with methanol.

Light microscopy. For light microscopy, smears from the insect gut, as well as flagellates from 7-8-day-old cultures, were fixed for 5 min with methanol and either stained with Giemsa stain and measured or incubated for $3 \mathrm{~min}$ in a phosphate-buffered saline solution with $0.1 \mu \mathrm{g}$ DAPI $\mathrm{ml}^{-1}$ (Sigma) at room temperature and examined with a confocal microscope (TCS SP2, AOBS; Leica). Flagellates from insects and cultures were measured using a Jenaval microscope with a calibrated micrometer (magnification $\times 1000$ ).

Electron microscopy. For transmission electron microscopy, flagellates collected from a 6-7-day-old culture were washed in $0.1 \mathrm{M}$ phosphate-buffered saline solution and fixed in $2.5 \%$ glutaraldehyde in $5 \mathrm{mM} \mathrm{HCl}, 0.1 \mathrm{M}$ cacodylate buffer for $1 \mathrm{~h}$ at room temperature, followed by at least $24 \mathrm{~h}$ at $4{ }^{\circ} \mathrm{C}$. Samples were post-fixed in $2 \%$ osmium tetroxide in the same buffer for $2 \mathrm{~h}$ at room temperature. After dehydration in a graded ethanol series, cells were embedded in Epon Araldite. Thin sections were stained with lead citrate and uranyl acetate and examined in a JEOL 1011 microscope. At least 50 specimens of each strain were measured from digital photographs using GRMEASURE software with a calibrated objective micrometer. 
Table 1. Summary of the species composition of examined and infected female biting midges from Mikulov, South Moravia (from 1998 to 2001) and Prague, Central Bohemia (from 1999 to 2002)

The prevalence of trypanosomatids in the gut is expressed as the number of infected midges divided by the total number of insects examined $(\%)$.

\begin{tabular}{|c|c|c|c|c|c|c|}
\hline Species & \multicolumn{2}{|c|}{ Mikulov } & \multicolumn{2}{|c|}{ Prague } & \multicolumn{2}{|c|}{ Total } \\
\hline C. (O.) festivipennis & 1206 & 7 & 330 & 1 & 1536 & 0.5 \\
\hline C. (O.) kibunensis & 27 & 1 & 380 & 7 & 407 & 2 \\
\hline C. (O.) minutissimus & 55 & 0 & 10 & 0 & 65 & 0 \\
\hline C. (O.) truncorum & 214 & 19 & 7 & 0 & 221 & 8.6 \\
\hline Total of all species & 1670 & 27 & 848 & 9 & 2518 & 1.4 \\
\hline
\end{tabular}

For scanning electron microscopy, flagellates were spotted onto poly Llysine-coated glass cover slips and fixed in $2 \% \mathrm{OsO}_{4}$ in $0.2 \mathrm{M}$ cacodylate buffer at $4{ }^{\circ} \mathrm{C}$ for $1 \mathrm{~h}$ and then washed in the same buffer at $4{ }^{\circ} \mathrm{C}$ for $30 \mathrm{~min}$. After dehydration, the cells were infiltrated with increasing concentrations of acetone, critical-point air-dried, sputtercoated with gold in a Polaron coater and examined using a JEOL 6300 scanning electron microscope.

DNA extraction for random amplified polymorphic DNA (RAPD). Total DNA from all seven flagellate strains obtained from the biting midges was isolated using a DNA tissue isolation kit (Roche) according to the manufacturer's instructions with the following modification; before adding proteinase $\mathrm{K}$, each sample was treated with lysis buffer and RNase A at $37^{\circ} \mathrm{C}$ for $1 \mathrm{~h}$. After this step, isolation was continued as described in the manual for the isolation of nucleic acids from mammalian tissue.

RAPD analysis. PCR amplicons were obtained with 12 decameric oligonucleotides (kit A, D, F; Operon Technologies): OPA 3 (AGTCAGCCAC), OPA 9 (GGGTAACGCC), OPA 10 (GTGATCGCAG), OPA 12 (TCGGCGATAG), OPD 3 (GTCGCCGTCA), OPD 8 (GTGTGCCCCA), OPD 11 (AGCGCCATTG), OPD 13 (GGGGTGACGA), OPD 18 (GAGAGCCAAC), OPF 1 (ACGGATCCTG), OPF 5 (CCGAATTCCC) and OPF 14 (TGCTGCAGGT).

Each $25 \mu \mathrm{l}$ RAPD reaction contained $5 \mathrm{ng}$ genomic DNA, $1.9 \mathrm{mM}$ $\mathrm{MgCl}_{2}$, 5 pmol oligonucleotide and $1 \mathrm{U}$ Taq polymerase in an appropriate buffer. The amplification cycle was $94^{\circ} \mathrm{C}$ for $1 \mathrm{~min}$, then 35 cycles at $94^{\circ} \mathrm{C}$ for $1 \mathrm{~min}, 38^{\circ} \mathrm{C}$ for $1 \mathrm{~min}$ and $72^{\circ} \mathrm{C}$ for $2 \mathrm{~min}$, and $72{ }^{\circ} \mathrm{C}$ for $10 \mathrm{~min}$. The RAPD products were separated by electrophoresis in ethidium bromide-stained $2 \%$ agarose gel. The reproducibility of RAPD profiles was high; however, the intensity of individual bands was subject to minor differences which may be due to small differences in DNA and primer concentrations.

DNA extraction, PCR and sequencing. Isolation of total DNA, PCR amplification and sequencing of the 18S rRNA gene have been described elsewhere (Votýpka et al., 2002). The 5S rRNA and SL RNA genes and the adjacent intergenic region were amplified using oligonucleotides under the conditions described by Podlipaev et al. (2004a). The gGAPDH gene was amplified using G3(F) and $\mathrm{G} 4 \mathrm{a}(\mathrm{R})$ oligonucleotides as described by Hamilton et al. (2004). Amplicons were gel-isolated (gel extraction kit; Qiagen) and were directly sequenced on an automated DNA sequencer (310 Genetic Analyzer; ABI Prism) using the BigDye 3.1 kit (Applied Biosystems).
Phylogenetic analysis. Four nuclear genes, 18S rRNA, 5S rRNA, SL RNA genes and the partial sequence of the gGAPDH gene, were used to establish the phylogenetic position of S. podlipaevi. Trees were computed from single genes as well as several concatenated alignments. Appropriate sequences of $18 \mathrm{~S}$ rRNA, 5S rRNA and SL RNA genes were aligned using the CLUSTAL_X program (Thompson et al., 1997). The GenBank accession numbers of analysed sequences and the composition of the final combined dataset are available as Supplementary Table S1 in IJSEM Online. Each alignment was manually checked and corrected; gaps and ambiguously aligned regions were omitted from further analysis. For the gGAPDH gene, we extended and modified the alignment of Hamilton et al. (2004). For the analyses presented here, three datasets were investigated: dataset1 [37 operational taxonomic units (OTUs); 2170 characters] is composed of concatenated 18S rRNA, 5S rRNA and SL RNA gene sequences. Dataset2 (75 OTUs; 861 characters) contains only gGAPDH nucleotide sequences. Dataset3 (37 OTUs; 3017 characters) combines the two previous datasets and comprises all available sequences. Phylogenetic analysis was performed using maximumparsimony (MP), maximum-likelihood (ML) and distance (D; LogDet-paralinear distance) methods with the PAUP $4.0 \mathrm{~b} 10$ (Swofford, 2001) and PHYML (Guindon \& Gascuel, 2003) software packages. ML trees were constructed using the general time reversible (GTR) model for nucleotide substitutions with $\gamma$-distribution in $8+1$ categories. All parameters $(\gamma$-shape, proportion of invariant sites, GTR parameters) were estimated from the particular dataset. Bootstrap analysis was computed in 300 replicates using the same model with $\gamma$-distribution in 4 categories and all parameters estimated from dataset. LogDet analysis was performed in PAUP (Swofford, 2001) using only variable positions in the dataset (735 nt), with bootstrap support calculated from 1000 replicates.

Nucleotide sequence accession numbers. The nucleotide sequences of the 18S rRNA, 5S rRNA, SL RNA and gGAPDH genes of three isolates (CER3, CER4 and CER7) of $S$. podlipaevi were identical and have been deposited in GenBank with accession numbers DQ394362, DQ394363, DQ394364 and DQ394365, respectively.

Experimental infections. Males and females of the biting midge, Culicoides (Monoculicoides) nubeculosus were obtained from Eric Denison and Karin Darpel (Department of Arbovirology, IAHPirbright laboratory, UK). Three-day-old adult biting midges were infected in batches of 300-400 by feeding them for $90 \mathrm{~min}$ through a parafilm membrane on a previously sterilized saline solution $(0.85 \% \mathrm{NaCl})$ heated to $37^{\circ} \mathrm{C}$ and containing six-day-old cultures of $10^{6} \mathrm{CER} 3$ promastigotes $\mathrm{ml}^{-1}$. In parallel, midges were also fed, 
in batches of 300-400, on a cotton pad saturated with a $15 \%$ sucrose solution containing cells of strain CER3. The midges were maintained in $250 \mathrm{ml}$ unwaxed paper cups at $100 \%$ relative humidity and fed daily on cotton soaked with $15 \%$ sucrose solution. The incubation temperature was $23^{\circ} \mathrm{C}$ [1-2 days post-infection (DPI)] and $18^{\circ} \mathrm{C}(3-20 \mathrm{DPI})$.

The midges were dissected at different time intervals (2-20 DPI). The location and number of protozoans in the gut and rectal ampulla of the midges were checked under a light microscope.

\section{RESULTS}

\section{Light microscopy}

As part of an extensive analysis of trypanosomatids parasitizing ceratopogonid flies in Southern Moravia and Prague performed between 1998 and 2002, a total of 2518 females belonging to C. (Beltranmyia) circumscriptus (101 specimens), Culicoides (Oecacta) festivipennis (1536 specimens), C. (O.) kibunensis (407 specimens), C. (O.) minutissimus (65 specimens), C. (O.) pictipennis (143 specimens), C. (O.) segnis (45 specimens) and C. (O.) truncorum (221 specimens) (see Table 1) were carefully dissected and their alimentary tracts were examined for flagellates. In total, four species of biting midges, $C$. festivipennis, C. kibunensis, C. pictipennis and C. truncorum, were found to be infected. Out of 36 positive females (the combined prevalence for all species was $\sim 1.5 \%$ ), a stable culture was successfully established for seven isolates (Table 2). The morphology of cells found in the smeared material showed that all flagellates were present in their hosts as promastigotes (Fig. la-b and data not shown). Importantly, after only several in vitro passages, the size of the cells of strains CER1, CER2, CER6 and CER8 doubled compared with the insect forms. The promastigotes of strains CER3, CER4 and CER7 remained unchanged. The strains that went through this significant cultivationinduced morphological transformation (strains CER1, CER2, CER6 and CER8) represent the species Herpetomonas ztiplika (Podlipaev et al., 2004b and Table 2).

Strains CER3, CER4 and CER7, however, retained their dimensions in culture and were studied further as they apparently represented a novel species. Their morphology in Giemsa-stained preparations is summarized in Table 2. The elongated promastigotes are equipped with a prominent flagellum, the length of which varies to some extent (Fig. 1a-b, d). The kinetoplast is positioned close to the anterior end of the cell (Fig. 1a-c). The morphology of strains CER3, CER4 and CER7 did not change even after several months of cultivation and the three strains were morphologically indistinguishable from each other.

\section{Electron microscopy}

Cells of strains CER3, CER4 and CER7 were examined by electron microscopy. The plasmalemma is underlaid by subpellicular microtubules with regular spacing (data not shown). The classical disc-shaped kinetoplast of standard 


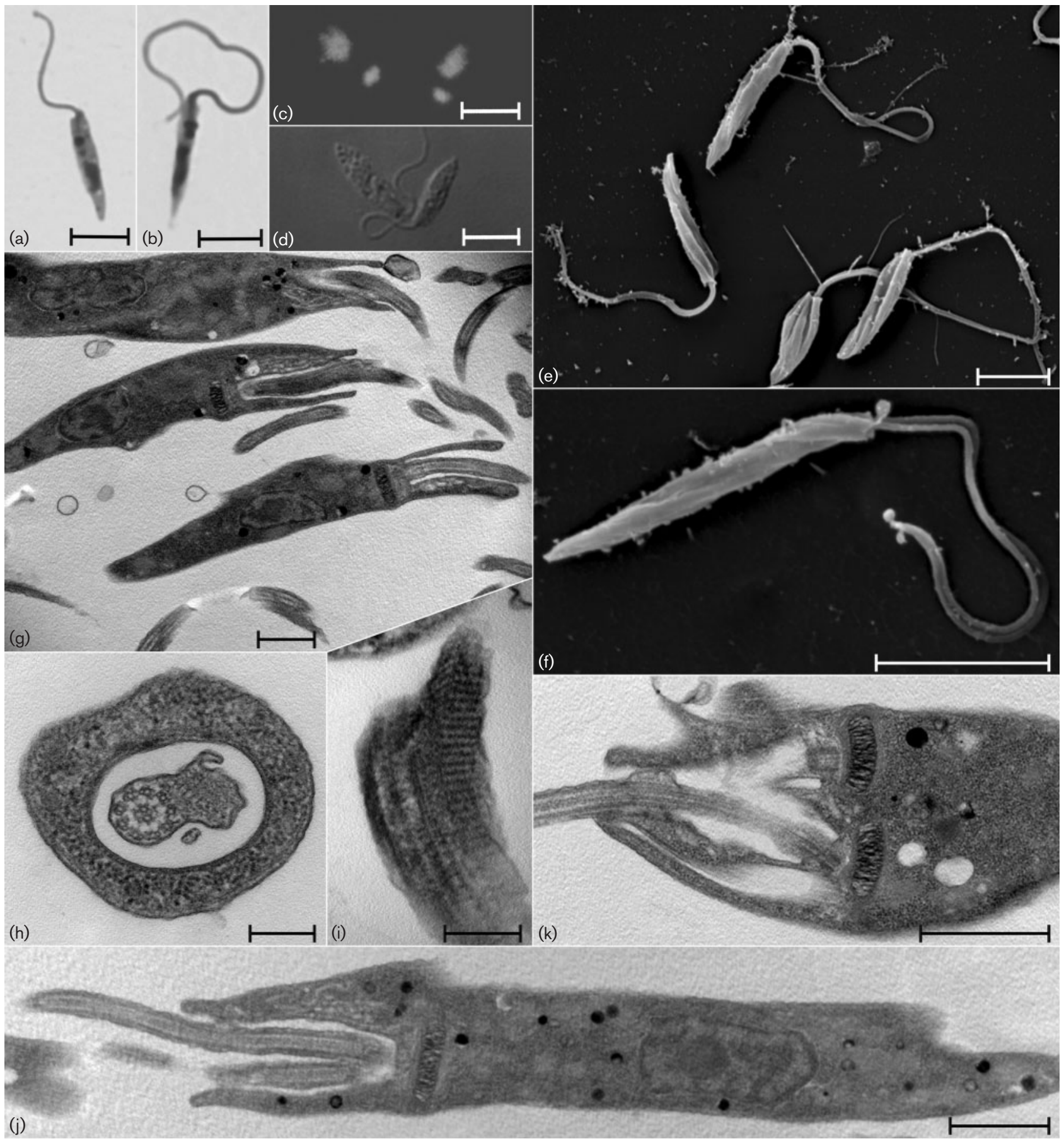

Fig. 1. Light $(a-b)$, confocal $(c-d)$, scanning $(e-f)$ and transmission ( $g-k)$ electron microscopy of cells of Sergeia podlipaevi sp. nov. ( $a, b)$ Giemsa-stained promastigotes in the smeared gut contents of biting midges. (c, d) DAPI-stained cultured promastigotes. (e, f) Twisted cells, some exhibiting a groove, with a prominent flagellum. (g) Longitudinal section of several elongated cells. (h) Flagellum cross-sectioned through the flagellar pocket which already bears a paraflagellar rod. (i) Longitudinal view of the paraflagellar rod revealing parallel filaments. (j) Longitudinal section of a dividing promastigote containing numerous acidocalcisomes. $(k)$ Anterior part of a promastigote at a late stage of division with two flagella and kinetoplasts. Bars, $5 \mu \mathrm{m}(\mathrm{a}-\mathrm{f}), 1 \mu \mathrm{m}(\mathrm{g}, \mathrm{j}-\mathrm{k})$ and $100 \mathrm{~nm}(\mathrm{~h}-\mathrm{i})$.

width and minicircle density is located next to the bottom of a deep flagellar pocket that may occupy a significant part of the cell body (Fig. 1g, j-k). The thickness of the kinetoplast is $0.205 \pm 0.015 \mu \mathrm{m}$ depending on the strain and differs significantly from the thickness of the kinetoplast of strains CER1, CER2, CER6 and CER8 belonging to H. ztiplika 
(Fig. 2). Based on the fact that the thickness of the disc is directly correlated with the size of kDNA minicircles (Lukeš \& Votýpka, 2000), the minicircles are estimated to be about $1.4 \mathrm{~kb}$ long. The kinetoplast-containing region is the only extended part of the thin elongated mitochondrion located on the periphery of the cell. In longitudinal sections, the nucleus is localized in the centre or close to the posterior end of the cell (Fig. 1g, j). Numerous acidocalcisomes are distributed throughout the anterior region of the cell (Fig. 1j). The flagellum is supported by a prominent paraflagellar rod (Fig. 1f, i) that can be seen in a crosssectioned flagellum even before it exits the flagellar pocket (Fig. 1h), although this supportive structure usually appears only in the external part of the flagellum (Bastin et al., 2000). Several filaments of the intermediate domain of the rod are shown in Fig. 1i. No bacterial endosymbionts were found in the cytoplasm, which is in agreement with the recent observation that symbiont-bearing species of trypanosmatids have a reduced paraflagellar rod (Gadelha et al., 2005).

Scanning electron micrographs revealed that the cell body is markedly twisted (Fig. 1e-f) and that the surface of some cells is striated with grooves (Fig. 1e).

\section{RAPD analysis}

RAPD analysis was performed using 12 oligonucleotides to test the sequence heterogeneity of all seven strains isolated from the biting midges (a representative RAPD gel is shown as Supplementary Fig. S1 in IJSEM Online). In total, 152 amplicons, ranging from $100 \mathrm{bp}$ to $4.0 \mathrm{~kb}$, were scored by size. The four strains assigned to H. ztiplika (CER1, CER2, CER6 and CER8) on the basis of morphometric analysis, kinetoplast thickness and culture-induced changes produced RAPD profiles that clearly differed from the other

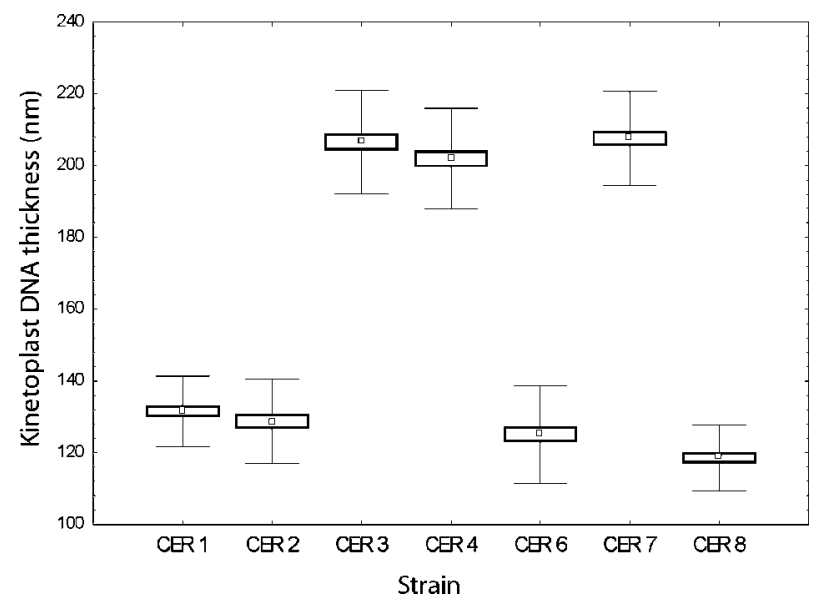

Fig. 2. Kinetoplast DNA thickness of three strains of Sergeia podlipaevi sp. nov. (CER3, CER4 and CER7) and four strains of Herpetomonas ztiplika (CER1, CER2, CER6 and CER8). The inner spread box represents $25-75 \%$, the outer spread line represents $\pm S D$. At least 50 cells of each strain were measured (STATISTICA, ANOVA). three isolates (CER3, CER4 and CER7). Only subtle intrastrain variations in the intensity of bands within the two groups were observed (data not shown). Thus, the RAPD analysis unambiguously separates strains CER3, CER4 and CER7 from the H. ztiplika strains, as seen on phylogenetic trees inferred from the dataset of all the RAPD patterns (see Supplementary Fig. S2 in IJSEM Online).

\section{Phylogenetic analysis}

We have sequenced the entire 18S, 5S and SL RNA genes and partial gGAPDH genes from the three strains, CER3, CER4 and CER7, representing the putative new flagellate species. The appropriate consensus sequences were used to construct single gene and concatenated alignments (see Supplementary Table S1 in IJSEM Online; datasets are available upon request). The final datasets $1-3$ contain members of all recognized trypanosomatid genera, as well as representatives of all major branches. In all three datasets analysed by MP, ML and LogDet, the bodonid Bodo saltans was used as an outgroup.

Trees computed based on dataset 1 and dataset 3 did not substantially differ and thus only those inferred from all concatenated genes (dataset3) and gGAPDH only-based trees (dataset2) are shown (Fig. 3). Both gGAPDH trees (ML and LogDet) show generally the same topology, with the exception of the positions of Leptomonas lactosovorans and Herpetomonas samuelpessoai. In all gGAPDH trees constructed, the novel species branched together with the sequence of an unidentified Phytomonas species as a sister group to the non-Trypanosoma trypanosomatids. The gGAPDH dataset clearly shows that the novel strains represent a new group of monoxenous trypanosomatids distinct from all species described so far.

The concatenated dataset (dataset3) leads to results similar to those obtained with the gGAPDH gene alone. Enriched sampling of the non-Trypanosoma trypanosomatids in dataset3 did not substantially help to establish the true position of the novel strains. As also found with dataset1 (18S, 5S and SL RNA genes), the basal nodes are not supported by bootstrap analyses and the positions of several clusters remain unstable. On the other hand, in all of the trees constructed, the novel strain branches together with the trypanosomatid 'EVA' (isolated from the dipteran Lutzomyia evansi in Venezuela) with $100 \%$ bootstrap support, regardless of the phylogenetic method or dataset used. However, the particular position of this clade is not well resolved; when using dataset 1 and ML criterion, the topology obtained does not differ from the dataset3-based ML tree shown in Fig. 3b. In both trees, basal nodes are not supported and the positions of particular clusters remain unresolved (data not shown). The ambiguity of dataset1, which is composed of the RNA coding genes, is well illustrated by parsimony analyses. Dataset 1 contains 477 parsimony-informative positions and leads to the construction of 508 equally parsimonious trees. The addition of the gGAPDH sequences (dataset3) increases the number of 
parsimony-informative positions to 728 and the number of equally parsimonious trees drops to 63 . However, in all these MP trees, S. podlipaevi appears in one cluster together with the trypanosomatid 'EVA', three Phytomonas isolates (Phytomonas serpens, Phytomonas sp. EM1 and Phytomonas sp. Hart1), Blastocrithidia triatoma and Leptomonas collosoma. The topology obtained is similar to that of the MP tree shown in Fig. 3b, left panel. The internal structure of this cluster differs slightly from tree to tree, but its composition remains stable. It should be noted that the instability of trees is also caused by the incompleteness of the available datasets (see Supplementary Table S1 for details). In ML trees, some Herpetomonas species (Herpetomonas muscarum, H. ztiplika, H. samuelpessoai and Herpetomonas megaseliae) appear within the above-mentioned cluster as a sister group to Phytomonas and Leptomonas, with S. podlipaevi, trypanosomatid 'EVA' and B. triatoma as a sister group to this cluster. However, this clade was again not supported by bootstrap analysis (see Fig. 3b, right panel).

In summary, S. podlipaevi clusters with the trypanosomatid 'EVA' with $100 \%$ bootstrap support in all of the analyses performed and both taxa seem to represent a new and separate cluster within the trypanosomatids. A robust position for this novel cluster cannot be obtained with the available dataset as topologies vary when different phylogenetic methods and datasets are applied.

\section{Experimental infection}

CER3-infected specimens were recovered from both feeding methods. Only females became infected after feeding through the membrane. When fed through the membrane, 18 out of 53 females were infected. When fed with the sugar solution, 55 out of 79 individuals became infected (females, 40/51; males, 15/28). The infection persisted for at least 20 days, with the promastigotes present in the midgut and hindgut. After 5 DPI, the promastigotes were present mainly in the Malpighian tubes, which corresponds to the localization observed previously during strain isolation from naturally infected insects. The ability of $S$. podlipaevi to develop in the intestine of biting midges representing two subgenera (two species of the subgenus Oecacta as natural hosts and a species of the subgenus Monoculicoides under experimental conditions) indicates a host range that extends over different subgenera of biting midges.

\section{Description of Sergeia gen. nov.}

Sergeia (Ser.ge.ia. Sergeia in honour of the eminent protozoologist and our dear friend Sergei Podlipaev, who passed away prematurely in 2004).

Monoxenous parasitic flagellate without bacterial endosymbionts. As determined from SL, 18S and 5S RNA and gGAPDH gene sequence-based analyses, the genus Sergeia is a member of the Trypanosomatidae, however, it cannot be associated with any valid genera of monoxenous or heteroxenous trypanosomatids. The type species is Sergeia podlipaevi.

\section{Description of Sergeia podlipaevi sp. nov.}

Sergeia podlipaevi (pod.li.pa.e.vi. podlipaevi in honour of the eminent protozoologist and our dear friend Sergei Podlipaev, who passed away prematurely in 2004).

The type strain was isolated from females of the biting midge Culicoides festivipennis Kieffer, 1914. Females of the biting midge Culicoides truncorum Edwards, 1939 are additional hosts. The parasite develops in the intestine of biting midges and is localized in the Malpighian tubes or in the abdominal midgut. Prevalence of the parasite is low, but may be up to several percent (Table 1). The mean sizes of the cells found in insects (calculated from strains CER3, CER4 and CER7) are $8.9 \pm 1.7 \mu \mathrm{m}$ [standard error (SE)] long, $2.1 \pm 0.2 \mu \mathrm{m}$ (SE) wide and have a flagellum length of $11.2 \pm 0.4 \mu \mathrm{m}$ (SE). In culture (values calculated from strains CER3, CER4 and CER7), cells have a mean length of $9.2 \pm 2.2 \mu \mathrm{m}$ (SE), width of $2.0 \pm 0.1 \mu \mathrm{m}$ (SE) and a flagellum length of $12.3 \pm 1.0 \mu \mathrm{m}$ (SE). There are no significant differences between the insect and culture forms. The nucleus is localized in the centre or close to the posterior end of the cell. Numerous acidocalcisomes are distributed throughout the anterior region. The flagellum is supported by a prominent paraflagellar rod present already within the flagellar pocket. The thickness of the kinetoplast is $0.205 \pm 0.015 \mu \mathrm{m}$ (SE). Cell morphology does not change, even after prolonged in vitro cultivation. No bacterial endosymbionts are present. Giemsa-stained slides of isolate CER3 (both insect and culture forms) have been deposited in the collection of the Department of Parasitology, Faculty of Science, Charles University, Prague, Czech Republic.

The type strain, CER3 (ICUL/CZ/2000/CER3), was isolated from biting midges trapped in the Milovicky forest, in the vicinity of Mikulov, Southern Moravia, Czech Republic, Europe. The type strain has been deposited in the collection of the Department of Parasitology, Faculty of Science, Charles University, Prague, and in the collection of the Institute of Parasitology, Centre for Biological Sciences, Academy of Sciences, Ceské Budějovice, Czech Republic.

\section{DISCUSSION}

Finding a trypanosomatid in a blood-sucking insect is always of particular interest for at least two reasons: (i) despite frequent examinations, monoxenous flagellates are very rarely encountered in blood-sucking hosts, and (ii) there is always a possibility that such a parasite may be equipped for the invasion of a vertebrate host. Several cases of possible transfer of monoxenic insect trypanosomatids into a mammalian host or tissue culture without an apparent preadaptation phase have been described (Jiménez et al., 1996; Podlipaev et al., 2004b; Santos et al., 2004). The fact that $S$. podlipaevi has been found in both blood-sucking females as well as in males feeding solely on 


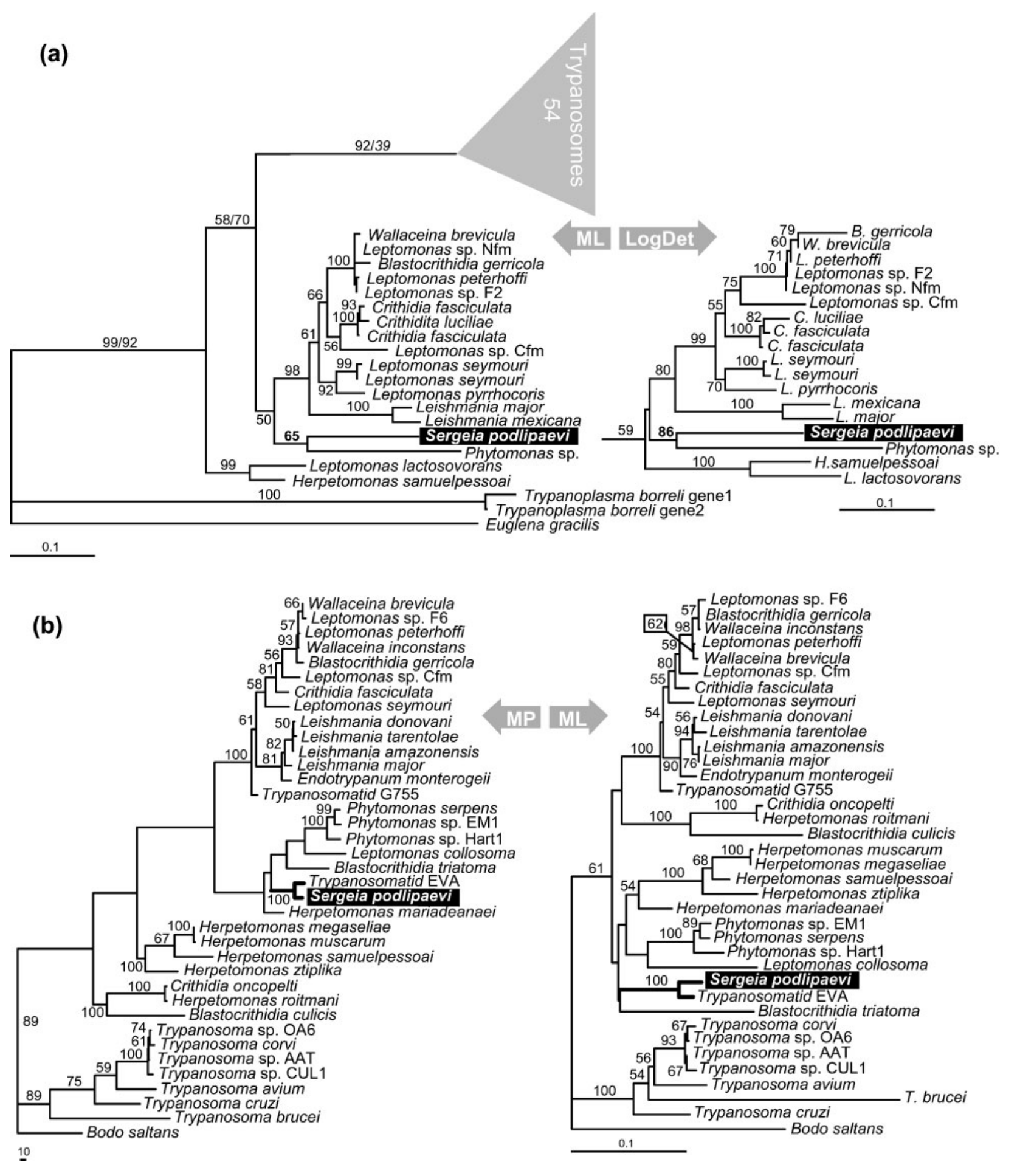

Fig. 3. (a) Phylogenetic trees as inferred from partial gGAPDH gene sequences. The figure shows topologies obtained by $M L$ (loglk =-14716.63587) (left) and LogDet-paralinear distances (from variable sites only) (right). The ML tree was computed using the GTR model for nucleotide substitutions with $\gamma$ distribution in $8+1$ categories as implemented in PHYML; all parameters were estimated from the dataset $(\gamma$-shape parameter $=0.523$; proportion of invariants $=0.166)$. Numbers above branches indicate bootstrap support (ML, 300 replicates; LogDet, 1000 replicates). Trees were rooted using the Euglena gracilis sequence as an outgroup. Bars represent 0.1 substitutions per site. (b) MP (left) and ML (loglk $=-17588.38484$ ) (right) phylogenetic trees as inferred from nucleotide sequences of the combined dataset (SSU $+S L+5 S+g G A P D H)$. Numbers above branches indicate appropriate bootstrap supports as computed from 500 replicates for MP and 300 replicates for ML. All ML parameters were estimated from the dataset (GTR with $\gamma$ distribution in $8+1$ categories as implemented in PHYML; $\gamma$-shape parameter $=0.237$; proportion of invariants $=0.000$ ). Bars represent total number of substitutions (left) and 0.1 substitutions per site (right). 
plant sap is strong evidence in favour of its monoxenous status.

Phylogenetic studies based on data from multiple genetic markers are now widely regarded as the 'gold standard' method for determining evolutionary relationships. In this study, we propose that phylogenetic topology with wide taxon coverage justifies a new genus and the description of a novel trypanosomatid species that does not fall into any known lineage. Due to the dearth of morphological features and to the extreme variability of those few available, attempts to compare the novel strains with previously described trypanosomatid species and genera using morphological characteristics were rendered futile. The promastigote-shaped cells imply an affiliation with members of the genera Leptomonas and Herpetomonas. The dimensions and motility of the promastigotes of strains CER3, CER4 and CER7, as well as the RAPD analysis, are sufficient to distinguish them from $H$. ztiplika, the only other trypanosomatid known to infect biting midges (Podlipaev et al., $2004 \mathrm{~b}$ ). The paraxial rod of the flagellum of $S$. podlipaevi is already present within the flagellar pocket, a feature that distinguishes it from other insect trypanosomatids for which fine structural data are available. However, we anticipate that this characteristic may also be found in unrelated flagellates and therefore would prefer to base our taxonomic decision on the nucleotide sequence data. To this end, we have amplified the 18S rRNA, SL RNA, 5S rRNA and gGAPDH genes, each of which has been previously shown to be informative for trypanosomatids (Fernandes et al., 1997; Lukeš et al., 1997; Dollet et al., 2000; Yurchenko et al., 2006). Phylogenetic analyses based on single-gene and concatenated alignments of these genes placed S. podlipaevi into a novel phylogenetic clade. The branching order relevant to the position of $S$. podlipaevi was generally not influenced by different outgroups and its separate branching was strongly supported.

Interestingly, in trees based on $18 \mathrm{~S}$ rRNA gene sequences, $S$. podlipaevi was joined by a closely related trypanosomatid, designated 'EVA' that was isolated from a nematocerous blood-sucking sand fly Lutzomyia evansi in Venezuela and for which a description and frozen stabilate are lacking (Fig. 3; Norris Rodriguez, personal communication). This nomen nudum nucleotide sequence seems to provide unexpected cross-continental evidence for a co-evolution of these parasites with their nematocerous hosts. The cluster composed of S. podlipaevi and strain 'EVA' branches off deeply and is not closely associated with any known trypanosomatid. The $18 \mathrm{~S}$ rRNA gene is able to delineate major clades within the trypanosomatid flagellates and individual branches of this tree have been suggested to represent the genus rank (Hollar et al., 1998).

In addition, the separate position of $S$. podlipaevi has been further corroborated by analysing three additional genes. Phylogenetic analysis of these single- and multi-copy genes, in combination with the moderately variable and hypervariable adjacent regions, provides discrimination between species or even strains. Taken together, sequences of all these genes support the establishment of a new genus. Since all currently recognized genera of monoxenous trypanosomatids are paraor polyphyletic in sequence-based analyses (Simpson et al., 2006), extension of any of them by additional species seems to be fruitless. The true diversity of trypanosomatid flagellates will be reflected only by an extensive taxonomic revision of the family Trypanosomatidae that accommodates sequencederived data.

\section{ACKNOWLEDGEMENTS}

We thank Eric Denison and Karin Darpel, Department of Arbovirology, IAH-Pirbright Laboratory, UK, for providing the Culicoides nubeculosus midges. We would like to express our thanks to Jiří Zídek for programming the GRMEASURE software. This research was supported by grants from the Ministry of Education of the Czech Republic (0021620828, LC06009, 6007665801 and 2B06129) and by the Grant Agency of the Czech Academy of Sciences (Z60220518).

\section{REFERENCES}

Abreu Filho, B. A., Dias Filho, B. P., Vermelho, A. B., Jankevicius, S. I., Jankevicius, J. V. \& dos Santos, R. L. (2001). Surface component characterization as taxonomic tools for Phytomonas spp. identification. Parasitol Res 87, 138-144.

Bastin, P., Pullen, T. J., Moreira-Leite, F. F. \& Gull, K. (2000). Inside and outside of the trypanosome flagellum: a multifunctional organelle. Microbes Infect 2, 1865-1874.

d’Ávila-Levy, C. M., Araújo, F. M., Vermelho, A. B., Branquinha, M. H., Alviano, C. S., Soares, R. M. A. \& dos Santos, A. L. S. (2004). Differential lectin recognition of glycoproteins in choanomastigoteshaped trypanosomatids: taxonomic implications. FEMS Microbiol Lett 231, 171-176.

Dollet, M., Sturm, N. R., Sanchéz-Moreno, M. \& Campbell, D. A. (2000). 5S ribosomal RNA gene repeat sequences define at least eight groups of plant trypanosomatids (Phytomonas spp.): phloemrestricted pathogens form a distinct section. J Eukaryot Microbiol 47, 569-574.

Fernandes, O., Teixeira, M. M. G., Sturm, N. R., Sousa, M. A., Camargo, E. P., Degrave, W. M. \& Campbell, D. A. (1997). Miniexon gene sequences define six groups within the genus Crithidia. J Eukaryot Microbiol 44, 535-539.

Fernandes, O., Santos, S. S., Cupolillo, E., Mendonca, B., Derre, R., Junqueira, A. C. V., Santos, L. C., Sturm, N. R., Naiff, R. D. \& other authors (2001). A mini-exon multiplex polymerase chain reaction to distinguish the major groups of Trypanosoma cruzi and T. rangeli in the Brazilian Amazon. Trans R Soc Trop Med Hyg 95, 97-99.

Gadelha, C., Wickstead, B., de Souza, W., Gull, K. \& Cunha-e-Silva, N. (2005). Cryptic paraflagellar rod in endosymbiont-containing kinetoplastid protozoa. Eukaryot Cell 4, 516-525.

Guindon, S. \& Gascuel, O. (2003). A simple, fast and accurate algorithm to estimate large phylogenies by maximum likelihood. Syst Biol 52, 696-704.

Hamilton, P. B., Stevens, J. R., Gaunt, M. W., Gidley, J. \& Gibson, W. C. (2004). Trypanosomes are monophyletic: evidence from genes for glyceraldehyde phosphate dehydrogenase and small subunit ribosomal RNA. Int J Parasitol 34, 1393-1404.

Hoare, C. A. \& Wallace, F. G. (1966). Developmental stages of trypanosomatid flagellates: a new terminology. Nature 212, 1385-1386. 
Hollar, L., Lukeš, J. \& Maslov, D. A. (1998). Monophyly of endosymbiont containing trypanosomatids: phylogeny versus taxonomy. J Eukaryot Microbiol 45, 293-297.

Jiménez, M. I., López-Vélez, R., Molina, R., Cañavate, C. \& Alvar, J. (1996). HIV-coinfection with a currently non-pathogenic flagellate. Lancet 347, 264-265.

Lukeš, J. \& Votýpka, J. (2000). Trypanosoma avium: novel features of the kinetoplast structure. Exp Parasitol 96, 178-181.

Lukeš, J., Jirků, M., Doležel, D., Král'ová, I., Hollar, L. \& Maslov, D. A. (1997). Analysis of ribosomal RNA genes suggests that trypanosomes are monophyletic. J Mol Evol 44, 521-527.

Merzlyak, E., Yurchenko, V., Kolesnikov, A. A., Alexandrov, K., Podlipaev, S. A. \& Maslov, D. A. (2001). Diversity and phylogeny of insect trypanosomatids based on small subunit rDNA genes: polyphyly of Leptomonas and Blastocrithidia. J Eukaryot Microbiol 48, 161-169.

Miranda, K., Docampo, R., Grillo, O. \& de Souza, W. (2004). Acidocalcisomes of trypanosomatids have species-specific elemental composition. Protist 155, 395-405.

Momen, H. (2001). Some current problems in the systematics of Trypanosomatids. Int J Parasitol 31, 640-642.

Podlipaev, S. A. (1990). Catalogue of world fauna of Trypanosomatidae (Protozoa). Proc Zool Inst Leningrad 144, 1-178.

Podlipaev, S. A. (2000). Insect trypanosomatids: the need to know more. Mem I Oswaldo Cruz 95, 517-522.

Podlipaev, S. A., Sturm, N. R., Fiala, I., Fernandes, O., Westenberger, S. J., Dollet, M., Campbell, D. A. \& Lukeš, J. (2004a). Diversity of insect trypanosomatids assessed from the spliced leader RNA and 5S rRNA genes and intergenic regions. J Eukaryot Microbiol 51, 283-290.

Podlipaev, S. A., Votýpka, J., Jirků, M., Svobodová, M. \& Lukeš, J. (2004b). Herpetomonas ztiplika n. sp. (Kinetoplastida: Trypanosomatidae): a parasite of the blood-sucking biting midge Culicoides kibunensis Tokunaga, 1937 (Diptera: Ceratopogonidae). J Parasitol 90, 342-347.

Poinar, G., Jr, \& Poinar, R. (2004). Paleoleishmania proterus n. gen., n. sp., (Trypanosomatidae : Kinetoplastida) from Cretaceous Burmese amber. Protist 155, 305-310.

Poinar, G., Jr, \& Poinar, R. (2005). Fossil evidence of insect pathogens. J Invertebr Pathol 89, 243-250.

Santos, D. O., Bourguignon, S. C., Castro, C. H., Silva, J. S., Franco, L. S., Hespanhol, R., Soares, M. J. \& Corte-Real, S. (2004). Infection of mouse dermal fibroblasts by the monoxenous trypanosomatid protozoa Crithidia deanei and Herpetomonas roitmani. J Eukaryot Microbiol 51, 570-574.

Santos, A. L. S., Abreu, C. M., Alviano, C. S. \& Soares, R. M. A. (2005). Use of proteolytic enzymes as an additional tool for trypanosomatid identification. Parasitology 130, 79-88.

Simpson, A. G. B., Lukeš, J. \& Roger, A. J. (2002). The evolutionary history of kinetoplastids and their kinetoplasts. Mol Biol Evol 19, 2071-2083.

Simpson, A. G. B., Stevens, J. R. \& Lukeš, J. (2006). The evolution and diversity of kinetoplastid flagellates. Trends Parasitol 22, $168-174$.

Stevens, J. R. (2001). One million insects - a lot of parasites? Trends Parasitol 17, 119-120.

Stevens, J. R., Noyes, H. A., Schofield, C. J. \& Gibson, W. (2001). The molecular evolution of Trypanosomatidae. Adv Parasitol 48, $1-56$.

Swofford, D. L. (2001). PAUP*. Phylogenetic analysis using parsimony ( ${ }^{\star}$ and other methods), version 4 . Sunderland, MA: Sinauer Associates.

Thompson, J. D., Gibson, T. J., Plewniak, F., Jeanmougin, F. \& Higgins, D. G. (1997). The CLUSTAL_X windows interface: flexible strategies for multiple sequence alignment aided by quality analysis tools. Nucleic Acids Res 25, 4876-4882.

Vickerman, K. (1994). The evolutionary expansion of the trypanosomatid flagellates. Int J Parasitol 24, 1317-1331.

Votýpka, J., Oborník, M., Volf, P., Svobodová, M. \& Lukeš, J. (2002). Trypanosoma avium of raptors (Falconiformes): phylogeny and identification of vectors. Parasitology 125, 253-263.

Wallace, F. G. (1966). The trypanosomatid parasites of insects and arachnids. Exp Parasitol 18, 124-193.

Wallace, F. G., Camargo, E. P., McGhee, R. B. \& Roitman, I. (1983). Guidelines for the description of new species of lower trypanosomatids. J Protozool 30, 308-313.

Westenberger, S. J., Sturm, N. R., Yanega, D., Podlipaev, S. A., Zeledón, R., Campbell, D. A. \& Maslov, D. A. (2004). Trypanosomatid biodiversity in Costa Rica: genotyping of parasites from Heteroptera using the spliced leader RNA gene. Parasitology 129, 537-547.

Yurchenko, V., Lukeš, J., Xu, X. \& Maslov, D. A. (2006). An integrated morphological and molecular approach to a new species description in the Trypanosomatidae: the case of Leptomonas podlipaevi n. sp., a parasite of Boisea rubrolineata (Hemiptera: Rhopalidae). J Eukaryot Microbiol 53, 103-111. 